\title{
NONRESONANCE CONDITIONS AND EXTREMAL SOLUTIONS FOR FIRST-ORDER IMPULSIVE PROBLEMS UNDER WEAK ASSUMPTIONS
}

\author{
DANIEL FRANCO ${ }^{1}$ and RODRIGO L. POUSO ${ }^{2}$
}

(Received 26 November, 1999; revised 12 April, 2000)

\begin{abstract}
In this work we shall study the existence of extremal solutions for an impulsive problem with functional-boundary conditions and weak regularity assumptions, not only on the right-hand side of the equation and on the functions that define the boundary conditions, but also on the impulse functions, which will be required to be nondecreasing, but not continuous as well, as is customary in the literature.

Moreover, in order to prove one of our results we shall study a general impulsive linear problem, giving a complete characterisation of resonance for it.
\end{abstract}

\section{Introduction}

The framework of impulsive differential equations has proved to be the most adequate for the modelling of processes with short time perturbations where change is assumed to occur instantaneously. These equations exhibit several new phenomena and pose a number of specific problems that cannot be treated with the usual techniques for ordinary differential equations (ODEs). There are many applications in different sciences for impulsive differential equations. We mention here a model for drug distribution $[11,12]$, the model of a single-species population with changes to important biological parameters $[5,17]$ and the impulsive stabilisation of a state which may not be an equilibrium point of the system (stabilisation of an inverted pendulum) [14].

In recent years many authors have considered different problems involving impulsive differential equations, and many types of techniques have been employed to deduce the existence of solutions: degree theory, topological transversality, fixed

\footnotetext{
'Departamento de Matemática Aplicada I, ETSI Industriales, UNED, Apartado de Correos 60149. 28080 Madrid, Spain; e-mail: dfranco@ind.uned.es.

${ }^{2}$ Departamento de Análise Matemática, Facultade de Matemáticas, Universidade de Santiago de Compostela, Campus Sur s/n, 15782 Santiago de Compostela, Spain.

(C) Australian Mathematical Society 2003, Serial-fee code 1446-1811/03
} 
point theorems, passing to the limit and the definition of a proper generalisation of the Poincaré map for the study of initial and periodic boundary value problems.

On the other hand, a recent trend in the study of the existence of solutions for ODEs is that of discontinuous nonlinearities. It is necessary to point out that the usual techniques we have mentioned before strongly depend on continuity, so one has to look for alternative ways to deal with this type of problem. The fundamental reference in this field we have followed is [10].

In practical situations impulse effects are constant over certain ranges of values, mainly when the impulses are used to keep a control over a determined system. Thus it is reasonable to consider discontinuous impulse functions, rather than continuous ones, to obtain more realistic models (for example, a model of medical drug distribution in which each patient receives either one or two doses per day, depending on his, or her, temperature).

However, to the best of our knowledge, we know of no paper on impulsive equations that considers discontinuous impulse functions.

As we said in the abstract we shall study the existence of extremal solutions for an impulsive problem with functional-boundary conditions when some of the classical regularity hypotheses over the functions that define the problem are removed. In particular, impulsive functions will be required to be nondecreasing but not continuous.

The results that we shall present extend and complement those in $[2,8-10,13]$ and are new even for the nonimpulsive case.

\section{Notation, definitions and preliminary results}

We shall study the impulsive functional-boundary value problem given by

$$
\begin{aligned}
& x^{\prime}(t)=f(t, x(t)) \quad \text { for a.a. } t \in J=[0,1], \\
& x\left(t_{k}^{+}\right)=I_{k}\left(x\left(t_{k}\right)\right), \quad k=1,2, \ldots, m, \\
& B(x(0), x)=0,
\end{aligned}
$$

where $0=t_{0}<t_{1}<\cdots<t_{m}<t_{m+1}=1$ is a fixed partition which corresponds to impulse effects.

REMARK. It is not difficult to prove that the results of the present work for (2.1) remain valid if we change the interval $J$ by an arbitrary compact real interval $[a, b]$ : a simple change of variable shows the equivalence between the two problems.

Following the standard notation, we consider the set

$$
\begin{aligned}
P C(J)= & \left\{x: J \rightarrow \mathbb{R} \mid x \text { is continuous in } J \backslash\left\{t_{1}, t_{2}, \ldots, t_{m}\right\}\right. \\
& \text { and there exist } \left.x\left(t_{k}^{-}\right)=x\left(t_{k}\right) \text { and } x\left(t_{k}^{+}\right), k=1,2, \ldots, m\right\},
\end{aligned}
$$


which is a Banach space with the norm $\|x\|=\sup \{|x(t)|: t \in J\}$.

In the space $P C(J)$ we consider the usual pointwise partial ordering: for $v, w \in$ $P C(J)$, we shall write $v \leq w$ when $v(t) \leq w(t)$ for all $t \in J$. In such a case, we define the interval $[v, w]=\{z \in P C(J): v \leq z \leq w\}$.

The general assumptions for problem (2.1) are listed below:

(F) (1) For every $x \in \mathbb{R}, f(\cdot, x)$ is Lebesgue measurable on $J$.

(2) For a.a. $t \in J, \limsup _{z \rightarrow x^{-}} f(t, z) \leq f(t, x) \leq \liminf _{z \rightarrow x^{+}} f(t, z)$ for all $x \in \mathbb{R}$.

(3) For every $R>0$ there exists $\psi \in L^{1}(J)$ such that $|f(t, x)| \leq \psi(t)$ for a.a. $t \in J$ and all $x \in \mathbb{R}$ with $|x| \leq R$.

(I) $I_{k}: \mathbb{R} \rightarrow \mathbb{R}$ is a nondecreasing function for each $k=1,2, \ldots, m$.

(B) $B: \mathbb{R} \times P C(J) \rightarrow \mathbb{R}$ is such that for every $\xi \in P C(J)$ we have that, for all $u \in \mathbb{R}, \lim \inf _{z \rightarrow u^{-}} B(z, \xi) \geq B(u, \xi) \geq \lim \sup _{z \rightarrow u^{+}} B(z, \xi)$. Moreover, for each $u \in \mathbb{R}$, the function $B(u, \cdot)$ is nonincreasing in $P C(J)$.

The type of functional-boundary conditions we use here follows the spirit of [9]. Note that the usual boundary conditions, such as initial or periodic conditions, are covered in the formulation of problem (2.1). For periodic boundary conditions it suffices to define $B(u, \xi)=u-\xi(1)$ for $(u, \xi) \in \mathbb{R} \times P C(J)$. Furthermore, not only the behaviour of the solution at the boundary is involved in the condition $B(x(0), x)=0$ and, for instance, a condition of the form $x(0)=\int_{1 / 3}^{1 / 2} x(s) d s$, can be studied in the frame of problem (2.1).

Let us denote $J_{0}=\left[0, t_{1}\right]$ and for $k=1,2, \ldots, m, J_{k}=\left(t_{k}, t_{k+1}\right]$. We define the following subspace of $P C(J)$ :

$$
\Omega=\left\{x \in P C(J): x_{\mid J_{k}} \in A C\left(J_{k}\right), k=0,1, \ldots, m\right\},
$$

where $A C(K)$ denotes the space of absolutely continuous functions in the interval $K$.

Now we define the concepts of a lower and upper solution for problem (2.1).

DEFINITION 2.1. A function $\alpha: J \rightarrow \mathbb{B}$ is a lower solution of problem (2.1) if $\alpha \in \Omega, f(\cdot, \alpha(\cdot))$ is measurable and

$$
\begin{array}{ll}
\alpha^{\prime}(t) \leq f(t, \alpha(t)) & \text { for a.a. } t \in J, \\
\alpha\left(t_{k}^{+}\right) \leq I_{k}\left(\alpha\left(t_{k}\right)\right), & k=1,2, \ldots, m, \\
B(\alpha(0), \alpha) \leq 0 . &
\end{array}
$$

A function $\beta: J \rightarrow \mathbb{R}$ is an upper solution of $(2.1)$ if $\beta \in \Omega, f(\cdot, \beta(\cdot))$ is measurable and

$$
\begin{aligned}
& \beta^{\prime}(t) \geq f(t, \beta(t)) \quad \text { for a.a. } t \in J, \\
& \beta\left(t_{k}^{+}\right) \geq I_{k}\left(\beta\left(t_{k}\right)\right), \quad k=1,2, \ldots, m, \\
& B(\beta(0), \beta) \geq 0 .
\end{aligned}
$$


Finally we say that $x$ is a solution of (2.1) if it is both a lower and an upper solution.

REMARK. A function $f: J \times \mathbb{R} \rightarrow \mathbb{R}$ is said to be superpositionally measurable or sup-measurable if $f(\cdot, x(\cdot))$ is measurable on $J$ whenever $x: J \rightarrow \mathbb{R}$ is a measurable function on $J$, see [1].

For instance, continuous, and even Carathéodory, functions are sup-measurable functions, so in that case, the condition " $f(\cdot, x(\cdot))$ is measurable" is unnecessary in Definition 2.1.

However, if $f$ only verifies (F), the composition $f(\cdot, x(\cdot))$ may not be a measurable function, even for continuous $x$ (see [8]).

REMARK. In [2] a particular case of the following problem is considered:

$$
\begin{array}{ll}
x^{\prime}(t)=q(x(t)) f(t, x(t)) & \text { for a.a. } t \in J=[0,1], \\
x\left(t_{k}^{+}\right)=I_{k}\left(x\left(t_{k}\right)\right), & k=1,2, \ldots, m, \\
B(x(0), x)=0, &
\end{array}
$$

where the function $q: \mathbb{R} \rightarrow(0, \infty)$ is such that $q, 1 / q \in L_{\text {loc }}^{\infty}(\mathbb{R})$ and

$$
\int_{0}^{ \pm \infty} \frac{d s}{q(s)}= \pm \infty
$$

Note that, although $f$ verifies condition (F), the product $q f$ may not fulfil it. However, this apparently more general situation can be studied as a particular case of problem (2.1).

Indeed, following the ideas of $[4,8]$, one can prove that $x \in \Omega$ is a solution of $(2.2)$ if and only if $\phi \circ x$ is a solution of

$$
\begin{array}{ll}
y^{\prime}(t)=f\left(t, \phi^{-1}(y(t))\right) & \text { for a.a. } t \in J=[0,1], \\
y\left(t_{k}^{+}\right)=\phi\left(I_{k}\left(\phi^{-1}\left(y\left(t_{k}\right)\right)\right)\right), & k=1,2, \ldots, m, \\
B\left(\phi^{-1}(y(0)), \phi^{-1} \circ y\right)=0, &
\end{array}
$$

where $\phi(x)=\int_{0}^{x} d s / q(s)$ for all $x \in \mathbb{R}$.

Moreover, since $\phi$ is an increasing homeomorphism from $\mathbb{B}$ onto $\mathbb{R}$, it can be proved that the function $(t, y) \in J \times \mathbb{R} \mapsto f\left(t, \phi^{-1}(y)\right)$ verifies condition (F), the impulse functions $\phi \circ I_{k} \circ \phi^{-1}$ are nondecreasing for $k=1,2, \ldots, m$ and $\tilde{B}(u, \xi)=$ $B\left(\phi^{-1}(u), \phi^{-1} \circ \xi\right)$ for $(u, \xi) \in \mathbb{R} \times P C(J)$ verifies condition (B).

If $\alpha$ and $\beta$ are, respectively, a lower and an upper solution of problem (2.1) and $\alpha \leq \beta$, we say that a solution $x$ of $(2.1)$ is maximal in $[\alpha, \beta]$ if for every solution $y \in[\alpha, \beta]$ of $(2.1)$ we have that $x \geq y$. We define the minimal solution in $[\alpha, \beta]$ by 
reversing the inequalities. When both minimal and maximal solutions in $[\alpha, \beta]$ exist, we call them the extremal solutions in $[\alpha, \beta]$.

Analogously, we say that $x_{*}$ and $x^{*}$ are, respectively, the minimal and the maximal solutions of (2.1), and we call them the extremal solutions, if they are solutions, and $x_{*}(t) \leq x(t) \leq x^{*}(t)$ for all $t \in J$, whenever $x$ is a solution of (2.1).

The following result is [8, Theorem 3.1], and we include it here for the convenience of the reader.

THEOREM 2.2. If $f: J \times \mathbb{R} \rightarrow \mathbb{R}$ verifies conditions (1) and (2) of (F) and, moreover, there exists $\psi \in L^{1}(J)$ such that $|f(t, x)| \leq \psi(t)$ for a.a. $t \in J=[0,1]$ and all $x \in \mathbb{R}$, then the initial value problem

$$
x^{\prime}(t)=f(t, x(t)) \quad \text { for a.a. } t \in J, \quad x(0)=x_{0},
$$

has extremal solutions for every $x_{0} \in \mathbb{R}$.

The following lemmas will be used in the proof of our main results.

LEMMA 2.3. Let $a, b \in \mathbb{R}, a \leq b$ and $h: \mathbb{R} \rightarrow \mathbb{R}$ be such that $h(a) \leq 0 \leq h(b)$ and $\liminf \operatorname{six}_{z-x^{-}} h(z) \geq h(x) \geq \lim \sup _{z \rightarrow x^{+}} h(z)$ for all $x \in[a, b]$.

Then there exist $c_{1}, c_{2} \in[a, b]$ such that $h\left(c_{1}\right)=0=h\left(c_{2}\right)$ and if $h(c)=0$ for some $c \in[a, b]$ then $c_{1} \leq c \leq c_{2}$, that is, $c_{1}$ and $c_{2}$ are, respectively, the smallest and largest of the zeroes of $h$ in $[a, b]$.

PROOF. We shall only prove the existence of $c_{1}$ since the existence of $c_{2}$ may be deduced using symmetric arguments.

If $h(a)=0$ then $c_{1}=a$. Assume $h(a)<0$. Since $\lim \sup _{z \rightarrow a^{+}} h(z) \leq h(a)<0$, there exists $\varepsilon>0$ such that $h(t)<0$ for all $t \in[a, a+\varepsilon]$. Set

$$
r=\sup \{t \in(a, b]: h(s)<0 \text { for all } s \in[a, t]\}
$$

and let us prove that $h(r)=0$.

If $h(r)<0$ we deduce (just as we did for $a$ in the last paragraph) that $h(t)<0$ for all $t \in\left[r, r+\varepsilon_{1}\right]$ for some $\varepsilon_{1}>0$, which contradicts the definition of $r$. Hence it must hold that $h(r) \geq 0$.

If $h(r)>0$, since $\liminf _{z \rightarrow r^{-}} h(z) \geq h(r)>0$, there exists $\varepsilon_{2}>0$ such that $h(t)>0$ for all $t \in\left[r-\varepsilon_{2}, r\right]$, which is impossible by the definition of $r$.

Finally, if $h(c)=0$ for some $c \in[a, b]$, we have $r \leq c$ by our choice of $r$. Thus we have to define $c_{1}=r$.

A set $S \subset P C(J)$ is said to be quasi-equicontinuous if for all $x \in S$ and $\varepsilon>0$ there exists $\delta>0$ such that $s, t \in J_{k}$ and $|s-t|<\delta$ implies $|x(s)-x(t)|<\varepsilon$.

The following result about the relatively compact sets in $P C(J)$ is a consequence of the Arzela-Ascoli theorem. The reader can find its proof in [13]. 
LEMMA 2.4. A set $S \subset P C(J)$ is relatively compact if and only if $S$ is bounded and quasi-equicontinuous.

The following result is an adaptation of [10, Theorem 1.4.7] for the spaces $P C(J)$ and $\Omega$.

LEMMA 2.5. Let $\alpha, \beta \in \Omega$ be such that $\alpha \leq \beta$ and $G:[\alpha, \beta] \rightarrow[\alpha, \beta]$ is a nondecreasing mapping. Moreover, suppose that there exists $v \in \Omega$ such that

$$
|G x(s)-G x(t)| \leq|v(s)-v(t)|, \quad s, t \in J_{k}, k=0,1, \ldots, m,
$$

for every $x \in[\alpha, \beta]$.

Then $G$ has a least fixed point $x_{*}$ in $[\alpha, \beta]$ and a greatest one, $x^{*}$. Furthermore, it is satisfied that

$$
x_{*}=\min \{x \in[\alpha, \beta]: G x \leq x\}, \quad x^{*}=\max \{x \in[\alpha, \beta]: G x \geq x\} .
$$

Proof. Let $\left\{x_{n}\right\}$ be a monotone sequence in $[\alpha, \beta]$. Since the operator $G$ is nondecreasing, $\left\{G x_{n}(t)\right\}$ is for each $t \in J$ a monotone sequence in $[\alpha(t), \beta(t)]$. Thus we define $x(t)=\lim _{n \rightarrow \infty} G x_{n}(t)$ for each $t \in J$.

From (2.3) it follows that for each $n \in \mathbb{N}$,

$$
\left|G x_{n}(s)-G x_{n}(t)\right| \leq|v(s)-v(t)|, \quad s, t \in J_{k}, k=1,2, \ldots, m .
$$

Therefore the sequence $\left\{G x_{n}\right\}$ is quasi-equicontinuous and bounded and, by Lemma 2.4, the sequence $\left\{G x_{n}\right\}$ converges in $P C(J)$ to $x$.

Now, (2.4) implies as $n \rightarrow \infty$ that

$$
|x(s)-x(t)| \leq|v(s)-v(t)|, \quad s, t \in J_{k}, k=0,1, \ldots, m .
$$

Since $v \in \Omega$, it follows from (2.5) that $x \in \Omega$.

In this situation, a straightforward application of [10, Theorem 1.2.2] yields the result.

\section{Linear problems: the resonance condition}

In this section we shall study the linear problem

$$
\begin{array}{ll}
x^{\prime}(t)=p(t) x(t)+q(t) & \text { for a.a. } t \in J, \\
x\left(t_{k}^{+}\right)=c_{k} x\left(t_{k}\right)+d_{k}, & k=1,2, \ldots, m, \\
x(0)=L(x)+\lambda, &
\end{array}
$$


where $p, q \in L^{1}(J), \lambda, c_{k}$ and $d_{k}$ are given real numbers and $L$ is a linear functional defined on the vector space $P C(J)$.

Note that for $c_{k}=1$ and $d_{k}=0, k=1,2, \ldots, m$, we have the corresponding nonimpulsive case, and for $L(u)=u(1)$ and $\lambda=0$ we have periodic boundary conditions.

In the following proposition we discuss completely the solvability of (3.1)-(3.3), obtaining a general characterisation of resonance. A first step in the study of the resonance of the periodic case is given in $[6,15,16]$.

In what follows, by $\sum_{s<t_{i}<t}$ we mean, as usual, $\sum_{\left(i: s<t_{i}<t\right)}$, and in the same way by $\prod_{s<t_{i}<t}$ we mean $\prod_{\left\{i: s<t_{i}<t\right\}}$.

Proposition 3.1. Let $p, q \in L^{1}(J), \lambda, c_{k}, d_{k} \in \mathbb{R}, k=1,2, \ldots, m$, and a linear mapping $L: P C(J) \rightarrow \mathbb{R}$ be fixed.

(a) (Resonance) If

$$
L\left(t \mapsto \prod_{t_{k}<t} c_{k} e^{\int_{0}^{t} p(s) d s}\right)=1,
$$

then the linear problem (3.1)-(3.3) is solvable if and only if

$$
-\lambda=L\left(t \mapsto \int_{0}^{t} \prod_{s<t_{k}<t} c_{k} e^{\int_{s}^{\prime} p(r) d r} q(s) d s\right)+L\left(t \mapsto \sum_{0<t_{k}<t} \prod_{u_{i}<t_{i}<t} c_{i} e^{\int_{t_{k}}^{t} p(r) d r} d_{k}\right),
$$

and, in such a case, the problem has infinitely many solutions, which are given by the expression

$$
x(t)=\tau \prod_{t_{k}<t} c_{k} e^{\int_{0}^{\prime} p(s) d s}+\int_{0}^{t} \prod_{s<t_{k}<t} c_{k} e^{\int_{s}^{\prime} p(r) d r} q(s) d s+\sum_{0<t_{k}<t} \prod_{t_{k}<t_{i}<t} c_{i} e^{\int_{t_{t}}^{\prime} p(r) d r} d_{k},
$$

where $\tau \in \mathbb{R}$.

(b) If condition (3.4) is violated, problem (3.1)-(3.3) has a unique solution given by (3.5) and

$$
\begin{aligned}
\tau= & \frac{\lambda+L\left(t \mapsto \int_{0}^{t} \prod_{s<i_{k}<1} c_{k} e^{\int_{1}^{t} p(r) d r} q(s) d s\right)}{1-L\left(t \mapsto \prod_{t_{k}<t} c_{k} e^{\int_{0}^{\prime} p(s) d s}\right)} \\
& +\frac{L\left(t \mapsto \sum_{0<t_{1}<t} \prod_{t_{k}<t_{i}<t} c_{i} e^{\int_{t_{k}}^{\prime} p(r) d r} d_{k}\right)}{1-L\left(t \mapsto \prod_{t_{k}<t} c_{k} e^{\int_{0}^{\prime} p(s) d s}\right)}
\end{aligned}
$$


PROOF. It is known that a function that satisfies (3.1)-(3.2) also satisfies the equality ([13, Theorem 1.4.1])

$$
\begin{aligned}
x(t)= & x(0) \prod_{t_{k}<t} c_{k} e^{f_{0}^{\prime} p(s) d s}+\int_{0}^{t} \prod_{s<t_{k}<t} c_{k} e^{\int_{s}^{t} p(r) d r} q(s) d s \\
& +\sum_{0<t_{k}<t t_{k}<t_{i}<t} \prod_{i} e^{\int_{t_{k}}^{\prime} p(r) d r} d_{k},
\end{aligned}
$$

for all $t \in J$.

Imposing the condition $x(0)=L(x)+\lambda$ and taking into account the linearity of $L$, one can deduce (a) and (b) by direct computation.

REMARK. Note that when we consider periodic boundary conditions (defining $L(x)=x(1)$ for $x \in P C(J)$ and $\lambda=0$ ), relation (3.4) becomes $\prod_{k=1}^{p} c_{k} e^{\int_{0}^{1} p(s) d s}=1$, and if, moreover, we consider the nonimpulsive case, that is, $c_{k}=1, d_{k}=0$, $k=1,2, \ldots, m$, we obtain $\int_{0}^{1} p(s) d s=0$.

REMARK. For the nonimpulsive case, corresponding to $c_{k}=1, d_{k}=0$, $k=1,2, \ldots, m$, the linear mapping $L$ is defined over the set of continuous functions on the interval $J$.

Therefore, if $L: \mathscr{C}(J) \rightarrow \mathbb{R}$ is a continuous functional, there exists a function of bounded variation $\eta: J \rightarrow \mathbb{R}$ such that $L(v)=\int_{0}^{1} v(t) d \eta(t)$ for all $v \in \mathscr{C}(J)$, where the integral is understood in the Riemann-Stieltjes sense.

For instance, the periodic conditions correspond to the function $\eta$, defined by $\eta(t)=0$ for $t \in[0,1)$ and $\eta(1)=1$.

Using this fact, condition (3.4) can be expressed more succinctly as

$$
\int_{0}^{1}\left(e^{\int_{0}^{t} p(s) d s}\right) d \eta(t)=1
$$

For our purposes we will require the following generalised maximum principle.

LEMMA 3.2. Let $p, q \in L^{\prime}(J), q(t) \geq 0$ for a.a. $t \in J, \lambda, c_{k}, d_{k} \in \mathbb{R}^{+}$, $k=1,2, \ldots, m$, and a linear nondecreasing mapping $L: P C(J) \rightarrow \mathbb{R}$ be fuxed.

If $x \in \Omega$ is a solution of (3.1)-(3.3) and condition

$$
L\left(t \mapsto \prod_{t_{k}<t} c_{k} e^{f_{0}^{t} p(s) d s}\right)<1
$$

is fulfilled, then $x(t) \geq 0$ for each $t \in J$. 
PROOF. Note that $x$ is explicitly given by (3.5) and (3.6). Hence, since $q$ and $d_{k}$, $k=1,2, \ldots, m$, are nonnegative, it suffices to show that $x(0) \geq 0$ to conclude our result.

Since $L$ is nondecreasing and $\lambda \geq 0$, by (3.3) we have that

$$
x(0)=L(x)+\lambda \geq L\left(t \mapsto x(0) \prod_{t_{k}<t} c_{k} e^{f_{0}^{t} p(s) d s}\right),
$$

and, since $L$ is linear, we obtain

$$
x(0)\left[1-L\left(t \mapsto \prod_{t_{k}<t} c_{k} e^{\int_{0}^{\prime} p(s) d s}\right)\right] \geq 0 .
$$

Therefore, in view of (3.8), we can conclude that $x(0) \geq 0$.

\section{Nonlinear problems (I): upper and lower solutions}

This section is devoted to proving the following existence result. Its proof is based on the generalised iterative technique described in [10]. However, unlike the usual way in which this technique is used (where the right-hand side of the differential equation is modified to construct the iterates), we modify the functional-boundary condition and keep the same right-hand side.

THEOREM 4.1. Let $\alpha$ and $\beta$ be, respectively, a lower and an upper solution of (2.1). Assume that $\alpha(t) \leq \beta(t)$ for all $t \in J$ and that conditions $(\mathrm{F}),(\mathrm{I})$ and $(\mathrm{B})$ are verified.

Then problem (2.1) has extremal solutions in $[\alpha, \beta]$.

REMARK. Note that continuity is not required over any of the elements which define problem (2.1). In particular, the impulse functions need not be continuous.

PROOF. Consider the mapping $G:[\alpha, \beta] \rightarrow[\alpha, \beta]$, defined as follows: for each $\eta \in[\alpha, \beta]$ define $G \eta$ as the minimal solution between $\alpha$ and $\beta$ of the impulsive initial value problem

$$
\left(P_{\eta}\right) \quad\left\{\begin{aligned}
x^{\prime}(t) & =f(t, x(t)), & & t \in J, \\
x\left(t_{k}^{+}\right) & =I_{k}\left(x\left(t_{k}\right)\right), & & k=1,2, \ldots, m, \\
x(0) & =\tau_{\eta}, & &
\end{aligned}\right.
$$

where $\tau_{\eta}$ is the minimal solution in $[\alpha(0), \beta(0)]$ of the implicit equation

$$
B\left(\tau_{\eta}, \eta\right)=0 \text {. }
$$


Note that, since $B(u, \cdot)$ is nonincreasing for every $u \in \mathbb{R}$, we have

$$
B(\beta(0), \eta) \geq B(\beta(0), \beta) \geq 0 \geq B(\alpha(0), \alpha) \geq B(\alpha(0), \eta),
$$

and hence, by assumption (B) and Lemma 2.3, we conclude that $\tau_{\eta}$ is well-defined.

The existence of the minimal solution of $\left(P_{\eta}\right)$ between $\alpha$ and $\beta$ can be proved by using Theorem 2.2 together with standard arguments with upper and lower solutions. It suffices to solve the problem "piecewise" over each interval $J_{k}, k=0,1, \ldots, m$, taking into account the following fact: if $x$ is the minimal minimal solution of $\left(P_{n}\right)$ between $\alpha$ and $\beta$ on the interval $\left[0, t_{k}\right]$, then $\alpha\left(t_{k}\right) \leq x\left(t_{k}\right) \leq \beta\left(t_{k}\right)$, and since $I_{k}$ is nondecreasing, we have that

$$
\alpha\left(t_{k}^{+}\right) \leq I_{k}\left(\alpha\left(t_{k}\right)\right) \leq I_{k}\left(x\left(t_{k}\right)\right) \leq I_{k}\left(\beta\left(t_{k}\right)\right) \leq \beta\left(t_{k}^{+}\right),
$$

thus the solution $x$ can be continued over the interval $\left[t_{k}, t_{k+1}\right]$, between $\alpha$ and $\beta$ and so on.

Note that if $x \in[\alpha, \beta]$ is a fixed point of $G$ then $x$ is a solution of $(2.1)$ in $[\alpha, \beta]$.

Let us prove that $G$ is nondecreasing in $[\alpha, \beta]$. Let $\eta_{1}, \eta_{2} \in[\alpha, \beta]$ be such that $\eta_{1} \leq \eta_{2}$. By definition, $\tau_{\eta_{i}}$ is the minimal solution in $[\alpha(0), \beta(0)]$ of $(4.1)$, replacing $\eta$ by $\eta_{i}, i=1,2$. Hence it is obviously true that if $\tau_{\eta_{1}}=\alpha(0)$ then $\tau_{\eta_{1}} \leq \tau_{\eta_{2}}$.

On the other hand, if $\tau_{\eta_{1}}>\alpha(0)$ then for every $\tau \in\left[\alpha(0), \tau_{\eta_{1}}\right)$ we have

$$
0>B\left(\tau, \eta_{1}\right) \geq B\left(\tau, \eta_{2}\right) \text {, }
$$

and we obtain $\tau_{\eta_{1}} \leq \tau_{\eta_{2}}$ again.

Then we can say that $G \eta_{2}$ is an upper solution of the initial value problem $\left(P_{\eta_{1}}\right)$ and $G \eta_{2} \geq \alpha$, so problem $\left(P_{\eta_{1}}\right)$ has a minimal solution between $\alpha$ and $G \eta_{2}$. Since the minimal solution of that problem between $\alpha$ and $\beta$ is $G \eta_{1}$, we have that $G \eta_{1} \leq G \eta_{2}$.

By condition (3) in (F) there exists $\psi \in L^{1}(J)$ such that

$$
\left|(G \eta)^{\prime}(t)\right| \leq \psi(t) \text { for a.a. } t \in J \text { and all } \eta \in[\alpha, \beta] .
$$

Now we define for $k=0,1, \ldots, m$ the function $v(t)=\int_{t_{k}}^{t} \psi(s) d s$ with $t \in J_{k}$. It is easy to see that $G$ satisfies (2.3) with this function $v$.

By Lemma $2.5, G$ has a minimal fixed point $x_{*} \in[\alpha, \beta]$ which, moreover, satisfies

$$
x_{*}=\min \{x \in[\alpha, \beta]: G x \leq x\} .
$$

Finally, if $x$ is a solution of $(2.1)$ in $[\alpha, \beta]$ then $B(x(0), x)=0$ and $x(0) \epsilon$ $[\alpha(0), \beta(0)]$, which implies that $x(0) \geq \tau_{x}$, by the definition of $\tau_{x}$, and hence $G x \leq x$. Thus, by (4.2), we conclude that $x * \leq x$, that is, $x$, is the minimal solution of (2.1) in $[\alpha, \beta]$.

To deduce the existence of the maximal solution of $(2.1)$ in $[\alpha, \beta]$ it suffices to redefine $G$ in the obvious way. 


\section{Nonlinear problems (II): global solvability}

Finding an upper and a lower solution for a problem of type (2.1) is not a simple matter in many practical situations. From this point of view it is useful to have sufficient conditions for the existence of upper and lower solutions. Moreover, it would be convenient to be able to be sure that one can find a lower solution, $\alpha$, and an upper solution $\beta$, such that $\alpha \leq \beta$ on $J$ and that all the solutions belong to $[\alpha, \beta]$.

In this sense, and following the spirit of [9, Proposition 6.1] and [10, Proposition 2.3.1], we present the following result.

THEOREM 5.1. Suppose that conditions (F), (I) and (B) are satisfied. Assume also that the following set of assumptions is fulfilled:

(a) There exist $p, q_{1}, q_{2} \in L^{1}(J)$ such that $p(t) x+q_{1}(t) \leq f(t, x) \leq p(t) x+q_{2}(t)$ for a.a. $t \in J$ and all $x \in \mathbb{R}$, and $f(\cdot, v(\cdot))$ is measurable whenever $v \in \Omega$.

(b) There exist constants $c_{k} \in(0, \infty)$ and $d_{1, k}, d_{2, k}, \in \mathbb{R} k=1,2, \ldots, m$, such that $c_{k} x+d_{1, k} \leq I_{k}(x) \leq c_{k} x+d_{2, k}$ for all $x \in \mathbb{R}$ and all $k=1,2, \ldots, m$.

(c) There exists a nondecreasing linear mapping $L: P C(J) \rightarrow \mathbb{R}$ and there exist constants $a \in(0, \infty)$ and $\lambda_{1}, \lambda_{2} \in \mathbb{R}$ such that

$$
a u-L(\xi)+\lambda_{1} \geq B(u, \xi) \geq a u-L(\xi)+\lambda_{2},
$$

for all $(u, \xi) \in \mathbb{R} \times P C(J)$.

If

$$
L\left(t \mapsto \prod_{t_{k}<t} c_{k} e^{f_{0}^{\prime} p(s) d s}\right)<a
$$

then problem (2.1) has extremal solutions among all its solutions.

Proof. The following impulsive problem

$$
\left\{\begin{aligned}
y^{\prime}(t) & =p(t) y(t)+q_{2}(t) & & \text { for a.a. } t \in J, \\
y\left(t_{k}^{+}\right) & =c_{k} y\left(t_{k}\right)+d_{2 . k}, & & k=1,2, \ldots, m, \\
y(0) & =(1 / a) L(y)-\lambda_{2} / a, & &
\end{aligned}\right.
$$

has a unique solution, $\beta$, by virtue of condition (5.1) and Proposition 3.1.

By assumption (a) we have that $\beta^{\prime}(t)=p(t) \beta(t)+q_{2}(t) \geq f(t, \beta(t))$ for a.a. $t \in J$. By condition (b), $\beta\left(t_{k}^{+}\right)=c_{k} \beta\left(t_{k}\right)+d_{2, k} \geq I_{k}\left(\beta\left(t_{k}\right)\right)$ for $k=1,2, \ldots, m$, and, finally, condition (c) implies that $B(\beta(0), \beta) \geq a \beta(0)-L(\beta)+\lambda_{2}=0$, and hence $\beta$ is an upper solution of (2.1). 
Similarly, if $\alpha$ denotes the unique solution of the problem

$$
\left\{\begin{aligned}
y^{\prime}(t) & =p(t) y(t)+q_{1}(t) & & \text { for a.a. } t \in J, \\
y\left(t_{k}^{+}\right) & =c_{k} y\left(t_{k}\right)+d_{1 . k}, & & k=1,2, \ldots, m, \\
y(0) & =(1 / a) L(y)-\lambda_{1} / a, & &
\end{aligned}\right.
$$

then $\alpha$ is a lower solution of (2.1).

If we denote $v \equiv \beta-\alpha$, we have that

$$
\begin{aligned}
v^{\prime}(t) & =p(t) v(t)+q_{2}(t)-q_{1}(t) \geq p(t) v(t) \text { for a.a. } t \in J, \\
v\left(t_{k}^{+}\right) & =c_{k} v\left(t_{k}\right)+d_{2, k}-d_{1 . k} \geq c_{k} v\left(t_{k}\right), \\
v(0) & =\frac{1}{a} L(v)+\frac{\lambda_{1}-\lambda_{2}}{a} \geq \frac{1}{a} L(v),
\end{aligned}
$$

so, by Lemma 3.2, we conclude that $\alpha \leq \beta$ on $J$.

Now, by virtue of Theorem 4.1, we can affirm that problem (2.1) has the extremal solutions in the interval $[\alpha, \beta]$.

Finally, one can prove that any solution of (2.1) belongs to $[\alpha, \beta]$. Indeed, let $x$ be a solution of (2.1) and call $w \equiv x-\alpha$. Assumptions (a), (b) and (c) allow us to use Lemma 3.2 to deduce that $w \geq 0$ in $J$, that is, $x \geq \alpha$ in $J$. Similar arguments show that $x \leq \beta$ in $J$.

REMARK. Under the conditions of Theorem 5.1 we can construct $a$ priori bounds on the solutions: it suffices to consider the functions $\alpha$ and $\beta$ which are defined in the proof.

Note that the explicit expression of $\alpha$ and $\beta$ is given by (3.5) and (3.6).

Theorem 5.1 is used to deduce the existence of global extremal solutions in the next example.

Consider the problem

$$
\begin{aligned}
& x^{\prime}(t)=\frac{1}{\sqrt{|t-1|}}+[x(t)]+\operatorname{sgn}(x(t))-\frac{1}{\sqrt{|t-1|}} x(t), \quad t \in J=[0,2], \\
& x\left(1^{+}\right)=[x(1)], \\
& k[x(0)]-\left[\int_{0}^{2} x(s) d s\right]-x(1)=0,
\end{aligned}
$$

where $k>0$ is a fixed number, $[s]$ denotes the greatest integer less than or equal to $s \in \mathbb{R}$ and

$$
\operatorname{sgn}(s)= \begin{cases}s /|s|, & \text { if } s \neq 0 \\ 0, & \text { if } s=0\end{cases}
$$


The assumptions of Theorem 5.1 are fulfilled if we consider

$$
p(t)=1-\frac{1}{\sqrt{|t-1|}}, \quad q_{1}(t)=-2+\frac{1}{\sqrt{|t-1|}}, \quad q_{2}(t)=1+\frac{1}{\sqrt{|t-1|}},
$$

for a.a. $t \in J$; constants $c_{1}=1, d_{1,1}=-1, d_{2,1}=0, a=k, \lambda_{1}=1, \lambda_{2}=-k$ and the linear nondecreasing mapping $L(v)=\int_{0}^{2} v(s) d s+v(1)$ for all $v \in \Omega$.

Now, by Theorem 5.1, we can say that if condition (5.1) is satisfied, then problem (5.2)-(5.4) has the extremal solution among all its solutions.

Note that in this case, condition (5.1) becomes

$$
\int_{0}^{2}\left(e^{\int_{0}^{s} p(r) d r}\right) d s+e^{\int_{0}^{1} p(s) d s}<k
$$

so problem (5.2)-(5.4) has global extremal solutions for sufficiently large values of $k>0$.

\section{What if the impulse functions are not nondecreasing?}

In [3] Cabada and Liz and in [7] Frigon and O'Regan show the existence of solutions for the periodic and initial value problem with impulses without assuming monotonicity conditions on functions $I_{k}$ (the authors required these functions to be continuous). In the following example we show that when some of the impulse functions are not nondecreasing the conclusion of Theorem 4.1 may fail to be valid.

Consider the following periodic problem:

$$
\begin{aligned}
& x^{\prime}(t)=3 x^{2 / 3}(t) \text { for a.a. } t \in[-1,1], \\
& x\left(0^{+}\right)=-x(0), \\
& x(-1)=x(1) .
\end{aligned}
$$

In this case the unique impulse effect is given by function $I(x)=-x$ for all $x \in \mathbb{R}$, which is not a nondecreasing function.

A lower solution of (6.1)-(6.3) is given by

$$
\alpha(t)= \begin{cases}0, & \text { if } t \in[-1,0], \\ (t-1)^{3}, & \text { if } t \in(0,1],\end{cases}
$$

and an upper solution is

$$
\beta(t)= \begin{cases}(t+1)^{3}, & \text { if } t \in[-1,0], \\ 0, & \text { if } t \in(0,1] .\end{cases}
$$

Note that $\alpha \leq \beta$, so every condition of Theorem 4.1 is verified except condition (I). 
Now we are going to prove that problem (6.1)-(6.3) has no minimal solution in $[\alpha, \beta]$, which will show that the conclusion of Theorem 4.1 is not valid in general for this type of impulse function.

Note that $x \equiv 0$ is a solution of (6.1)-(6.3) in $[\alpha, \beta]$ and if $y \in[\alpha, \beta]$ is another solution we have two possibilities: there exists $t_{0} \in(-1,0)$ such that $y\left(t_{0}\right)>0$ or $t_{0} \in(0,1)$ such that $y\left(t_{0}\right)<0$.

Assume $t_{0} \in(-1,0)$ such that $y\left(t_{0}\right)>0$. Since the problem

$$
v^{\prime}(t)=3 v^{2 / 3}(t) \text { for all } t \in\left[t_{0}, 0\right], \quad v\left(t_{0}\right)=y\left(t_{0}\right),
$$

has a unique increasing solution, then $y(0)>0$ which implies that $y\left(0^{+}\right)=-y(0)<0$, so there exists $\varepsilon>0$ such that $y(t)<0$ for $t \in(0, \varepsilon)$.

Reasoning in a symmetric way it can be proved that if there exists $t_{0} \in(0,1)$ such that $y\left(t_{0}\right)<0$ then there exists $\varepsilon>0$ such that $y(t)>0$ for $t \in(-\varepsilon, 0)$.

Hence we have proved that no nontrivial solution of $(6.1)-(6.3)$ in $[\alpha, \beta]$ can be minimal, because it is not less than or equal to the trivial solution over the whole interval $[-1,1]$. On the other hand, we cannot say that $x \equiv 0$ is the minimal solution because there exist nontrivial solutions in $[\alpha, \beta]$ : consider, for instance, the function $y$ defined by

$$
y(t)= \begin{cases}(t+1)^{3}, & \text { if } t \in[-1,0], \\ (t-1)^{3}, & \text { if } t \in(0,1] .\end{cases}
$$

On the other hand, when some of the functions $I_{k}$ are discontinuous and not nondecreasing, not even the existence of a solution can be assured, as the following simple example shows:

$$
\begin{aligned}
& u^{\prime}(t)=0, \quad t \in[0,2], \\
& u\left(1^{+}\right)=I(u(1)), \\
& u(0)=u(2),
\end{aligned}
$$

with $I(x)=1$ if $x \in(\infty, 0)$, and $I(x)=-1$ if $x \in[0, \infty)$. Functions $\alpha=-2$ and $\beta=2$ are respectively lower and upper solutions of problem (6.4) but this problem is unsolvable.

\section{Acknowledgements}

This work was partially supported by DGESIC, project PB97-0552-C02, and by Xunta de Galicia, project XUGA20701B98. 


\section{References}

[1] J. Appell and P. P. Zabrejko, Nonlinear superposition operators (Cambridge University Press, 1990).

[2] A. Cabada and E. Liz, "Discontinuous impulsive differential equations with nonlinear boundary conditions", Nonlinear Anal. 28 (1997) 1491-1497.

[3] A. Cabada and E. Liz, "Boundary value problems for higher order ODEs with impulses", Nonlinear Anal. 32 (1998) 775-786.

[4] A. Cabada and R. L. Pouso, "On first-order discontinuous scalar differential equations", Nonlinear Studies 6 (1999) 161-170.

[5] L. H. Erbe, H. I. Freedman, X. Z. Liu and J. H. Wu, "Comparison principles for impulsive parabolic equations with applications to models of single-species growth", J. Austral. Math. Soc. Ser. B 32 (1991) 382-400.

[6] D. Franco and J. J. Nieto, "A new maximum principle for impulsive first-order problems", Internat. J. Theoret. Phys. 37 (1998) 1607-1616.

[7] M. Frigon and D. O'Regan, "Existence results for first-order impulsive differential equations", $J$. Math. Anal. Appl. 193 (1995) 96-113.

[8] E. R. Hassan and W. Rzymowski, "Extremal solutions of a discontinuous differential equation", Nonlinear Anal. 37 (1999) 997-1017.

[9] S. Heikkilä, "First-order discontinuous differential equations with functional boundary conditions", in Advances in nonlinear dynamics, Stability Control Theory Methods Appl. 5, (Gordon and Breach, Amsterdam, 1997) 273-281.

[10] S. Heikkilä and V. Lakshmikantham, Monotone iterative techniques for discontinuous nonlinear differential equations (Marcel Dekker, New York, 1994).

[11] E. Kruger-Thiemer, "Formal theory of drug dosage regiments, I", J. Theoret. Biol. 13 (1966) 212-235.

[12] E. Kruger-Thiemer, "Formal theory of drug dosage regiments, II", J. Theoret. Biol. 23 (1969) 169-190.

[13] V. Lakshmikantham, D. D. Bainov and P. S. Simeonov, Theory of impulsive differential equations (World Scientific, Singapore, 1989).

[14] X. Liu and A. Willms, "Impulsive stabilizability of autonomous systems", J. Math. Anal. Appl. 187 (1994) 17-39.

[15] E. Liz and J. J. Nieto, "Positive solutions of linear impulsive differential equations", Commun. Appl. Anal. 2 (1998) 565-571.

[16] J. J. Nieto, "Basic theory for nonresonance impulsive periodic problems of first order", J. Math. Anal. Appl. 205 (1997) 423-433.

[17] Y. V. Rogovchenko, "Nonlinear impulse evolution systems and applications to population models", J. Math. Anal. Appl. 207 (1997) 300-315. 